\title{
Erratum to: Preoperative carbohydrate loading for elective surgery: a systematic review and meta-analysis
}

\author{
Lun Li $\cdot$ Zehao Wang $\cdot$ Xiangji Ying • \\ Jinhui Tian · Tiantian Sun - Kang Yi • \\ Peng Zhang $\cdot$ Zhang Jing $\cdot$ Kehu Yang
}

(C) Springer 2012 precede "Croatia $(n=1)$ ". In Table 1 , the name of the country following Faria [37] should be Brazil, not the Czech Republic. The authors apologize for the errors.

In the "Characteristics of included trials" section, "Brazil $(n=1)$ " should follow "the Czech Republic $(n=1)$ " and

The online version of the original article can be found under doi:10.1007/s00595-012-0188-7.

L. Li $\cdot$ Z. Wang $\cdot$ X. Ying $\cdot$ J. Tian $\cdot$ K. Yi $\cdot$ P. Zhang $\cdot$

Z. Jing $(\bowtie) \cdot K$. Yang

Evidence-Based Medicine Center, School of Basic Medical

Sciences, Lanzhou University, Dong Gang West Road No. 199,

Lanzhou 730000, Gansu, China

e-mail: guo19880101@126.com

L. $\mathrm{Li}$

e-mail: guo19880101@163.com

Z. Wang

e-mail: athlete.dg@gmail.com

$X$. Ying

e-mail: yingxjii@163.com

J. Tian

e-mail: tjh996@163.com

K. Yi

e-mail: yikang04@126.com

P. Zhang

e-mail: zhp-118@163.com
K. Yang

e-mail: kehuyangebm2006@126.com

L. Li $\cdot$ K. Yi $\cdot$ P. Zhang $\cdot$ K. Yang

The First Clinical College of Lanzhou University,

Lanzhou 730000, Gansu, China

Z. Wang $\cdot$ X. Ying

The Second Clinical College of Lanzhou University,

Lanzhou 730000, Gansu, China

T. Sun

Shanghai Jiao-Tong University School of Medicine Ren-ji

Hospital, Shanghai 20000, China

e-mail: suntt2005@126.com

Z. Jing

Institute of Science and Technology Information of Gansu, Lanzhou 730000, China 